\title{
Detecting epidemiological relevance of adenoid hypertrophy, rhinosinusitis, and allergic rhinitis through an Internet search
}

\author{
Yingchao Yang ${ }^{1} \oplus \cdot$ Xinyi $^{\mathrm{Li}^{1}} \cdot$ Qiang $\mathrm{Ma}^{1} \cdot$ Zhihui Fu$^{1} \cdot \mathrm{Kaiming} \mathrm{Su}^{1} \mathbb{C}$
}

Received: 17 March 2021 / Accepted: 13 May 2021 / Published online: 9 June 2021

(c) The Author(s), under exclusive licence to Springer-Verlag GmbH Germany, part of Springer Nature 2021

\begin{abstract}
Purpose This study aimed to detect the epidemiological relevance between adenoid hypertrophy (AH) and rhinosinusitis, and AH and allergic rhinitis (AR) through an Internet search.

Methods Internet search query data from January 2011 to December 2019 in China were retrieved from the Baidu Index (BI). Spearman's correlation coefficients were used to detect the correlation among the search volumes of AH, rhinosinusitis, and AR. We also collected search data from the first 5 months of 2020, when quarantine was implemented in China due to the coronavirus disease 2019 epidemic. Then, we compared the search data to those obtained during the same period in 2019 to assess the effects of isolation on AH and AR.

Results Statistically significant relevance was found between the search variations of AH and rhinosinusitis during 2011$2019(R=0.643, P<0.05)$. However, the relationship between AH and AR was weak $(R=-0.239, P<0.05)$ and that between rhinosinusitis and $\mathrm{AR}(R=-0.022, P>0.05)$ was not relevant. The average monthly search volume of AH and rhinosinusitis had a strong correlation $(R=0.846, P<0.01)$, but AH and AR and rhinosinusitis and AR were not correlated $(R=-0.350$, $P>0.05 ; R=-0.042, P>0.05$, respectively). AH and rhinosinusitis search volumes decreased consistently during the first 5 months of 2020 (isolation), whereas that for AR increased during January-February.

Conclusion AH had an epidemiological relationship with rhinosinusitis, which was not consistent with AR. The decrease in public gathering effectively reduced the morbidities of AH and rhinosinusitis but not those of AR.
\end{abstract}

Keywords Adenoid hypertrophy $\cdot$ Allergic rhinitis $\cdot$ Baidu index $\cdot$ COVID-19 $\cdot$ Rhinosinusitis $\cdot$ Search volume

\section{Introduction}

Adenoid hypertrophy (AH) is the most common cause of obstructive sleep apnea (OSA) in children. The pathogenesis of AH is not yet clear, but it is related to many factors, such as smoking, seasonal allergies, and respiratory infections [1]. The relationship between $\mathrm{AH}$ and rhinosinusitis has been confirmed in previous studies, and therapies aimed at AH effectively treat rhinosinusitis [2, 3]. However, the information concerning the relationship between $\mathrm{AH}$ and allergic rhinitis (AR) is not consistent. Several scholars believe that

Kaiming Su

021china@sina.com

1 Shanghai Key Laboratory of Sleep Disordered Breathing, Department of Otorhinolaryngology, Head \& Neck Surgery, The Sixth People's Hospital Affiliated to Shanghai Jiaotong University, Yishan Road 600, Xuhui District, Shanghai 200233, China
AR triggers AH [4], whereas others believe that the relationship is irrelevant or that the two are negatively related $[5,6]$.

Traditional epidemiology surveillance relies on clinical data. Currently, advanced online networks provide people with access to medical knowledge, and patients can gain a basic understanding of their diseases using computers and mobile phones. Such search behaviors can be recorded and converted into search query data using an Internet search engine. Systems based on search engines can provide realtime information about diseases and have good congruence with traditional healthcare surveillance systems [7]. Studies have detected seasonal patterns of influenza epidemics, depression, restless leg symptomatology, and sleep-disordered breathing using Internet search query data [8-11]. Specific terms searched by netizens reflect their demands, and investigating Internet data could be a powerful complement to monitoring systems.

Baidu is one of the world's largest search engines. Since Google's withdrawal from China in 2010, Baidu has become 
China's main search engine. The 2018 study by Cao et al. [12] demonstrated that the Baidu Index (BI) of AR and its related search terms showed obvious seasonal patterns, which were consistent with epidemiological survey results; AR also had a significant correlation with the actual pollen concentration and outpatient visits. This again shows that the BI could be an indicator for the incidence of diseases.

In December 2019, several hospitals in Wuhan City, Hubei Province, identified unexplained pneumonia cases in persons with a history of exposure to the South China Seafood Market. The patients were subsequently confirmed to have severe acute respiratory syndrome coronavirus 2 (SARS-CoV-2) infection, a strain of coronavirus that causes coronavirus disease 2019 (COVID-19). The number of newly discovered cases increased exponentially, and this rapidly spreading novel virus has become the most widespread global pandemic that humans have encountered in the past century. In response to COVID-19, the Chinese government locked down Wuhan from January 23, 2020, to April 8,2020 , and urged the whole nation to remain isolated at home or in the hospital. Thus, an opportunity was provided to investigate the effect of isolation on diminishing the morbidities of $\mathrm{AH}$ and rhinosinusitis.

This study used Internet search data provided by BI to verify that $\mathrm{AH}$ and rhinosinusitis shared similar seasonal patterns that were irrelevant to AR and analyzed the effects of different degrees of isolation on reducing the morbidities of the three diseases during the COVID-19 epidemic in China.

\section{Methods}

\section{Data collection}

Internet search engine query data for the terms "adenoid hypertrophy," "rhinosinusitis," and "allergic rhinitis" in Chinese from January 2011 to December 2019 were retrieved from the BI. The first 5 months of 2020 data were added to our study's timeframe to observe the effect of isolation by comparing search volumes from the same period for 2019 and 2020. Since 2020 was a leap year, we excluded the data from February 29 to ensure that the amount of data was consistent with that of 2019.

The daily search volume in our study was defined as the average number of people searching for the term per day, that is, the average daily search volume for the term. It was obtained from the BI (http://index.baidu.com) and then added to the monthly search volume. Following data normalization against the total search volume, results are displayed on a scale from 0 to $100 \%$. Individual values over time were calculated by dividing each monthly volume by the yearly search volume; thus, the data are presented as percentages.

\section{Data analysis}

The main variables examined were the percentages of monthly search volume for $\mathrm{AH}$, rhinosinusitis, and AR in Chinese from January 2011 to May 2020. Statistical analyses were performed using SPSS Statistics version 26.0 (IBM Corp., Armonk, NY, USA). Continuous variables were tested for normality and then analyzed using the nonparametric Kruskal-Wallis test, as needed. Spearman's correlation coefficients were used to analyze the correlation between quantitative variables.

\section{Results}

A total of 339 monthly values, in percentage form, were obtained: AH $(n=113)$, rhinosinusitis $(n=113)$, and AR $(n=113)$.

The tendency charts of the three search terms from January 2011 to December 2019, and before the outbreak of the COVID-19 epidemic, are presented in Fig. 1, which shows that the two trendlines representing $\mathrm{AH}$ and rhinosinusitis searches coincided well. Furthermore, the seasonal variations of the two trendlines were statistically relevant $(R=0.643, P<0.05)$, while the relationship between $\mathrm{AH}$ and AR was weak $(R=-0.239, P<0.05)$. The searches for AR significantly increased from August to September, whereas the searches for $\mathrm{AH}$ were at their minimum during this period. Also shown are the irrelevant fluctuations between the rhinosinusitis and AR searches $(R=-0.022, P>0.05)$.

The average monthly search volumes for the three terms during the 9-year timeframe are presented in Fig. 2. The highest and lowest average monthly search volumes for $\mathrm{AH}$ were in December and September, respectively. The search volume for AH had one small and one big valley in February and September, respectively. The searches for rhinosinusitis showed a similar trend, which statistically correlated with AH $(R=0.846, P<0.01)$, but the trend was more gradual from August to October. The curve of the search for AR had two peaks: April-May and August-September. As searches for AR increased in June, the searches for AH decreased. Such a contrast appeared again when the searches for AR decreased and searches for AH increased simultaneously in September. No correlation was found between $\mathrm{AH}$ and AR or rhinosinusitis and AR $(R=-0.350, P>0.05 ; R=-0.042$, $P>0.05$, respectively).

The search volumes for all three terms decreased during the first 5 months of 2020 compared with those months in 2019 (Fig. 3). This phenomenon was abnormal because the total search volume for $\mathrm{AH}$ and rhinosinusitis had increased 
a

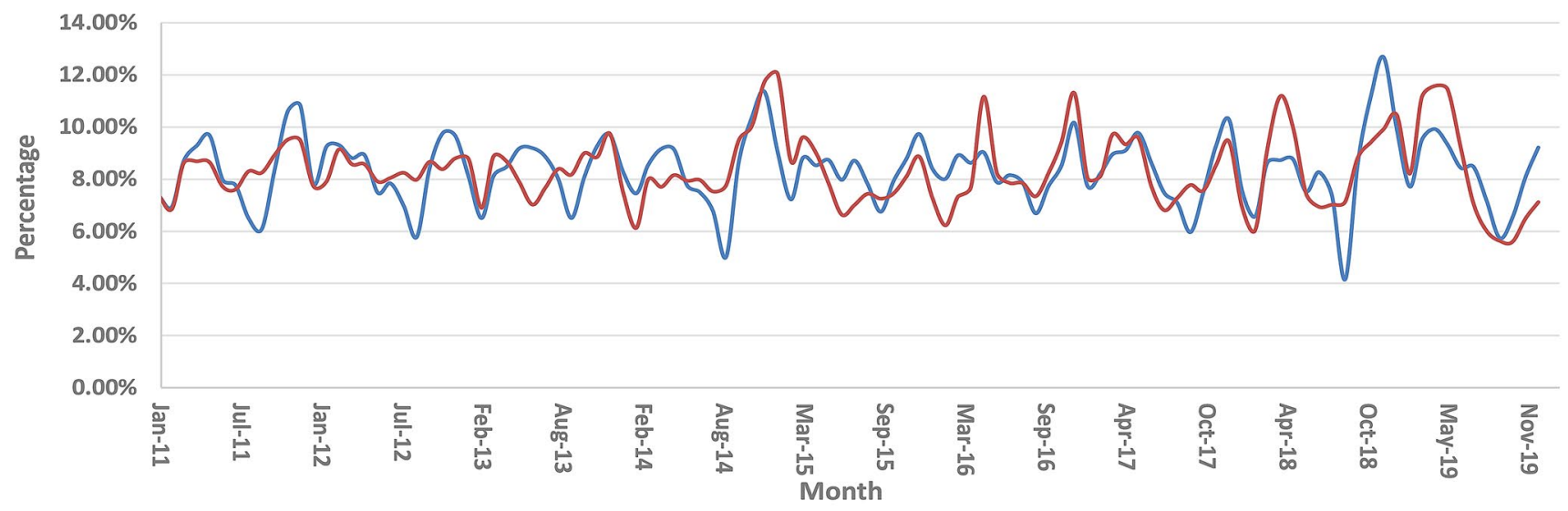

b

- $\mathrm{AH} \longrightarrow$ Rhinosinusitis

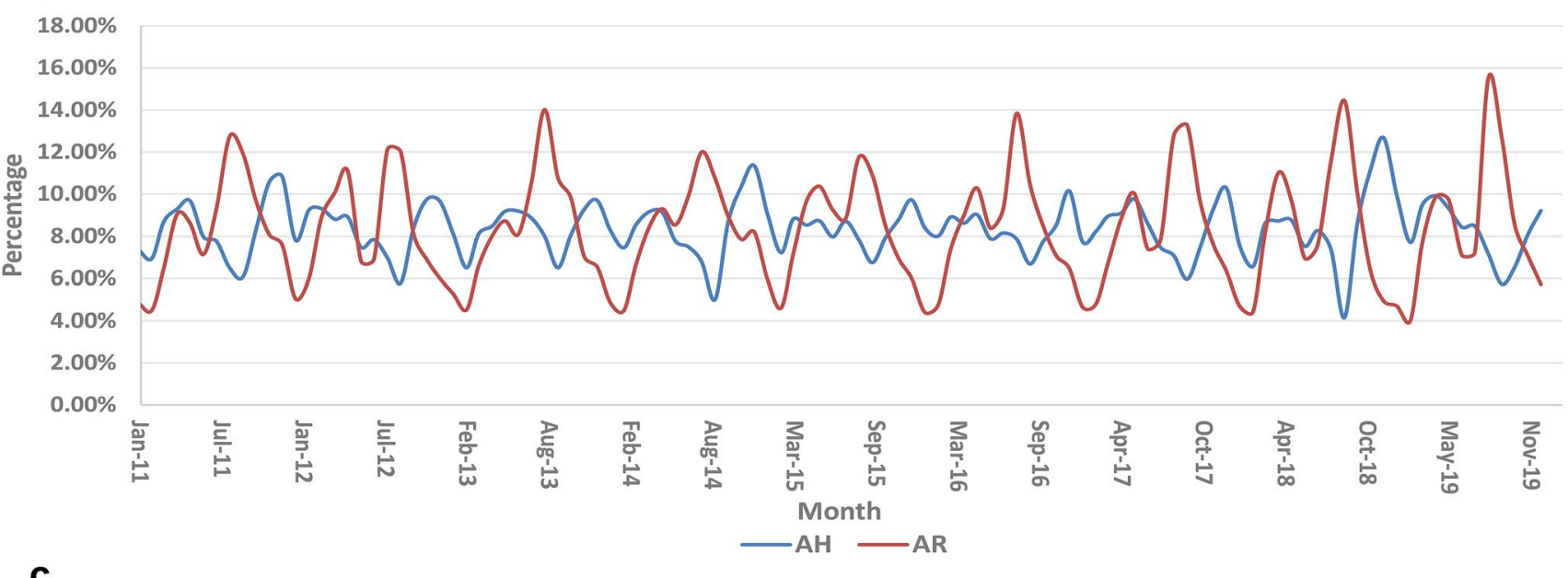

C

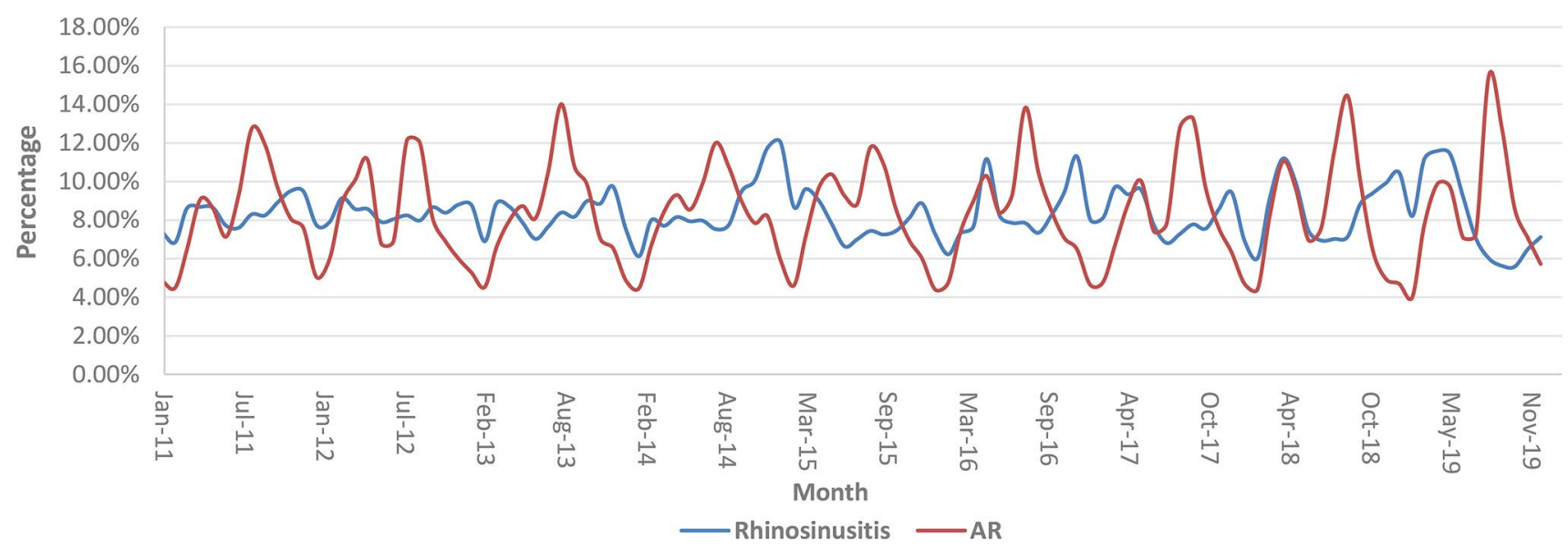

Fig. 1 Monthly search volume (in percentage) for a AH and rhinosinusitis, b $\mathrm{AH}$ and $\mathrm{AR}$, and $\mathbf{c}$ rhinosinusitis and $\mathrm{AR}$ during 2011-2019. The search variations for $\mathrm{AH}$ and rhinosinusitis are significantly relevant $(R=0.643, P<0.05)$ and show similar patterns in fluctuation. The search variations for AH and AR are not consistent $(R=-0.239, P<0.05)$, with opposite fluctuations to a certain extent. The search variations for rhinosinusitis and AR are not related $(R=-0.022, P>0.05)$. $A H$ adenoid hypertrophy, $A R$ allergic rhinitis 


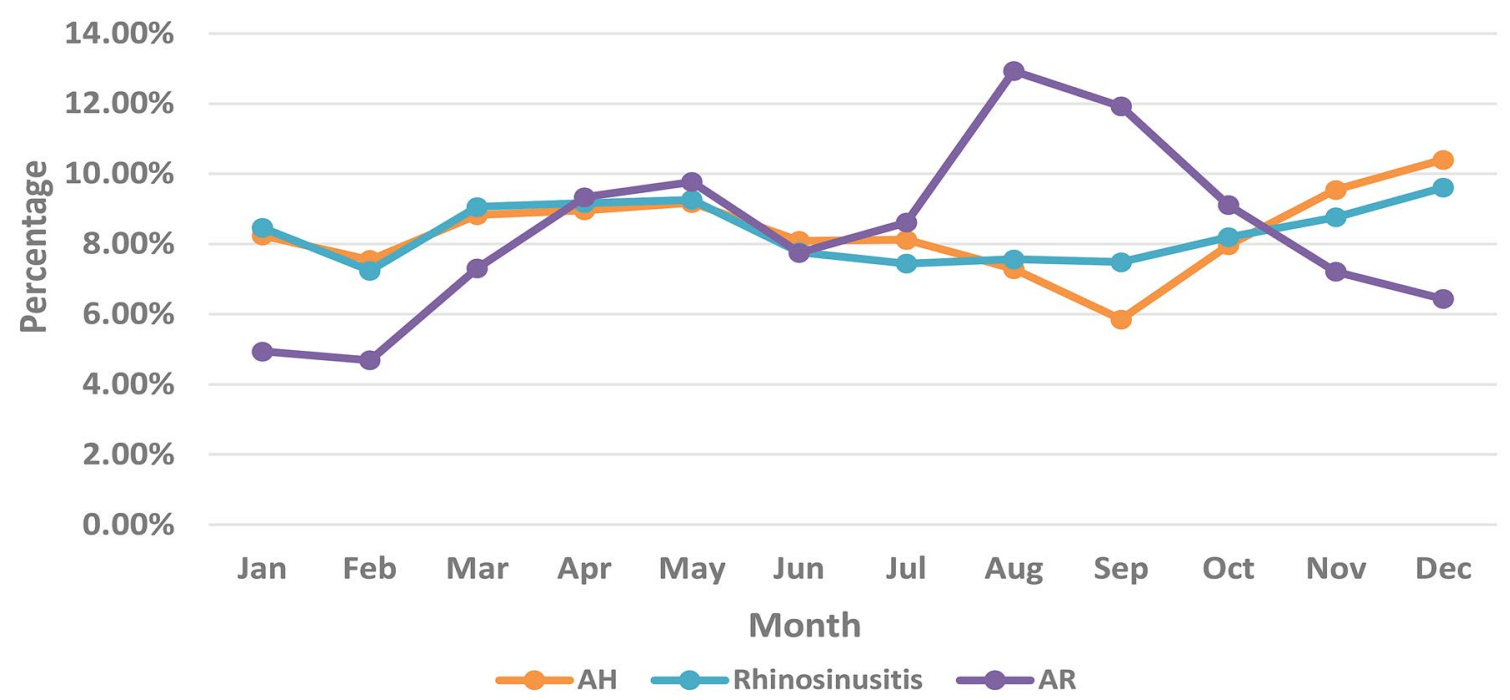

Fig. 2 Average monthly search volume for $\mathrm{AH}$, rhinosinusitis, and $\mathrm{AR}$. The search variations of $\mathrm{AH}$ and rhinosinusitis have a strong correlation $(R=0.846, P<0.01)$. They both decline from December to February and from May to September and then increase from February to May and from September to December. The search vari-

annually since 2011. Although the search for AR growth was not as significant as for $\mathrm{AH}$ and rhinosinusitis, it had also grown steadily. The $\mathrm{AH}$ and rhinosinusitis search volumes in the first 5 months of 2020 decreased by $41.21 \%(P<0.001)$ and $54.31 \%(P<0.001)$, respectively, from the search volumes during the same period in 2019. However, the rate of decline for AR was only $28.08 \%(P<0.001)$, which was significantly lower than those for $\mathrm{AH}$ and rhinosinusitis. The searches for AR from January to February in 2020 were even higher than those in 2019.

\section{Discussion}

The present study investigated the epidemiological relevance of AH, rhinosinusitis, and AR using an Internet database. Our results showed that the search volume of $\mathrm{AH}$ and rhinosinusitis varied in a statistically consistent manner. This consistency was also present during the period of isolation in China in response to the COVID-19 pandemic. However, the search volumes for $\mathrm{AH}$ and $\mathrm{AR}$, and rhinosinusitis and $\mathrm{AR}$ did not show statistical consistency; hence, further research is needed to determine their relationship.

The use of Internet data for analyzing infectious disease epidemics has become an important topic in the prevention and control of infectious diseases. Online surveillance tools based on Google Trends have been explored in recent years. Since Baidu is the largest search engine in China, its search queries could represent the population's needs, particularly in regions with a high Internet user rate. The BI use has ations of $\mathrm{AH}$ and $\mathrm{AR}$, and rhinosinusitis and $\mathrm{AR}$ are not correlated $(R=-0.350, P>0.05 ; R=-0.042, P>0.05$, respectively). Although searches for AR increase from June to August, those for AH decline. Although searches for AR decrease from September to December, those for $\mathrm{AH}$ increase. $A H$ adenoid hypertrophy, $A R$ allergic rhinitis

been shown to significantly correlate with the incidence of the human immunodeficiency virus (HIV)/acquired immunodeficiency syndrome (AIDS) [7]. Previous studies [7-11, 13] based on search engine query data focused on the regularities of specific diseases and building analysis models to predict their outbreaks. Our study analyzed three different diseases, AH, rhinosinusitis, and AR, and investigated their interrelationships.

The association between $\mathrm{AH}$ and $\mathrm{AR}$ is controversial; some scholars believe that AR triggers $\mathrm{AH}$ [4] and $\mathrm{AH}$ increases AR severity and prolongs disease duration [14]. Several scholars suggest that children with both AH and AR may be more sensitive to specific allergens, such as dust mites and molds [15, 16]. However, Ameli et al. [17] showed that large adenoids might be associated with the absence of allergy, and Eren et al. [5] also believe that AH and AR are negatively correlated. Studies have also shown that adenoidectomy did not notably benefit most children with $\mathrm{AH}$ and AR $[18,19]$. In this study, based on the analyses of 9 years of search data, we could not find statistically consistent variations between the search volumes of $\mathrm{AH}$ and $\mathrm{AR}$, which did not support AR being one of the risk factors of $\mathrm{AH}$.

The adenoid is an important immune organ in children; the volume of the adenoid increases after birth and starts reducing after 6-7 years. Previous studies have confirmed the existence of a strong relationship between $\mathrm{AH}$ and rhinosinusitis. For example, AH continuously affects the occurrence and development of rhinosinusitis in several ways, including causing posterior nasal obstruction, hindering nasal drainage, and constantly releasing pathogenic 
a

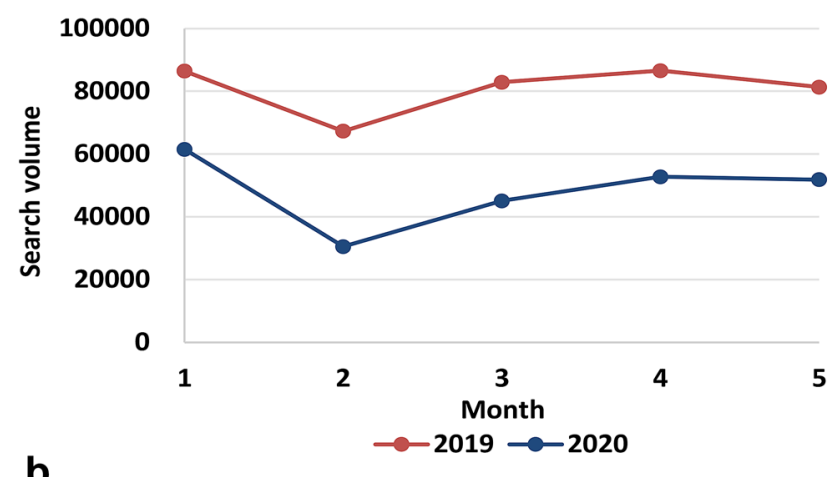

b

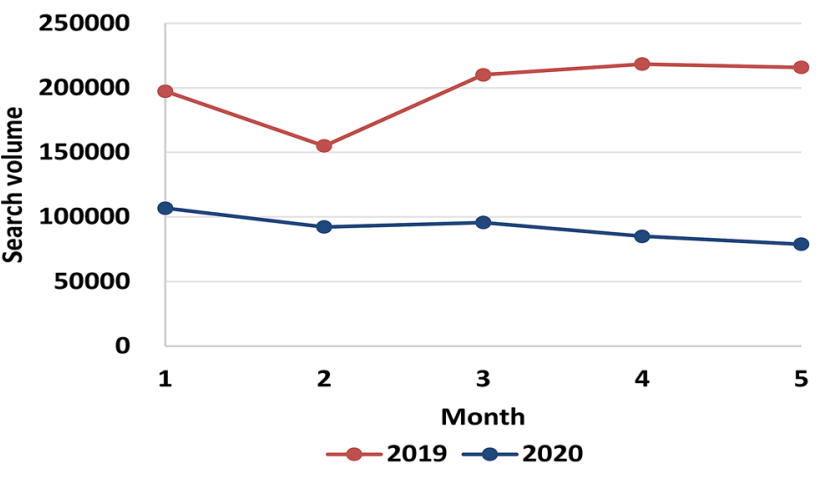

C

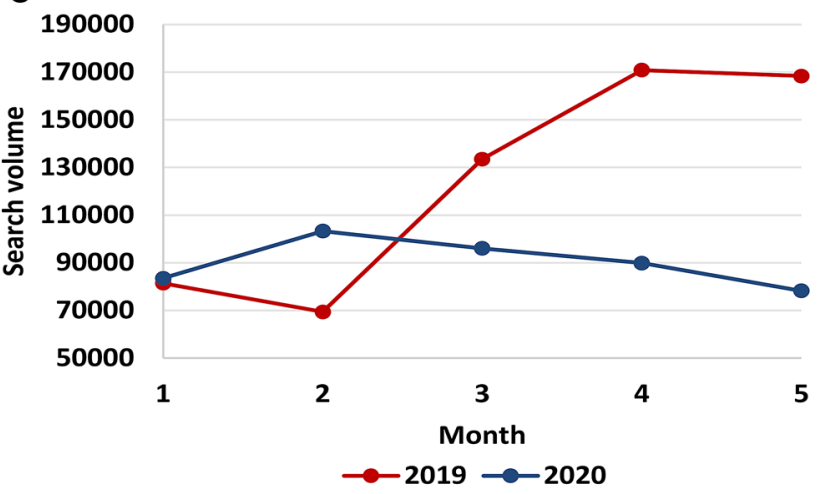

Fig. 3 Comparison of the search volume for $\mathbf{a} \mathrm{AH}$, b rhinosinusitis, and c AR during the first 5 months of 2019 and 2020. AH and rhinosinusitis search volumes in the first 5 months of 2020 decrease by $41.21 \%$ and $54.31 \%$, respectively, compared with the same period in 2019. The total search volume for AR decreases by only $28.08 \%$ and even increase in January and February. $A H$ adenoid hypertrophy, $A R$ allergic rhinitis

bacteria [20-23]. Rhinosinusitis, in turn, can lead to AH through inflammatory stimulation. Antibiotics usually relieve the symptoms of adenoid hypertrophy, although adenoidectomy is recommended as a first-line surgical treatment for chronic sinusitis [24-26]. In this study, the search volume of AR and rhinosinusitis also shared similar seasonal patterns, which may be explained by the seasonal variations of viral infections, as this was one of their mutual risk factors.
After the outbreak of COVID-19 in China, schools were closed down and classes were held online until May 2020. This could explain the decline in morbidity of AH and rhinosinusitis to some extent. Furthermore, after public activities resumed, a reexamination of some children with $\mathrm{AH}$ in our department revealed that their adenoid volume was reduced. Dave et al. [27] found that, approximately 3 weeks after enacting a statewide shelter-in-place order, the average number of cumulative cases decreased by approximately $53.5 \%$, again demonstrating the effect of isolation in reducing pathogen transmission. Considering the decline in morbidity during winter and summer holidays, we assumed that avoiding gatherings of people can reduce the morbidity of $\mathrm{AH}$ and rhinosinusitis. However, it was not very effective for AR.

Another important measure is wearing facemasks. Studies have confirmed the effect of facemasks in preventing infections. Brienen et al. [28] concluded that the extensive use of facemasks could help delay an influenza pandemic and reduce the reproduction number. Stutt et al. [29] reported the results of two mathematical models and concluded that the use of facemasks combined with physical distancing or periods of lockdown might be a way of managing the COVID19 pandemic and enabling resumption of economic activity. Hence, the use of facemasks was strictly mandated in China during the COVID-19 pandemic. Since facemasks can block various pathogenic particles, it will be interesting to investigate if they contribute to decreasing $\mathrm{AH}$ morbidities.

Our study has several limitations. First, since rhinosinusitis includes acute rhinosinusitis, chronic rhinosinusitis, and other kinds of rhinosinusitis, it would have been ideal to conduct the study by accounting for these different groups. Unfortunately, the terms "acute rhinosinusitis" and "chronic rhinosinusitis" were not included in BI due to reduced search volume, making a study of rhinosinusitis subtypes difficult. Second, the symptoms of these three conditions have some overlaps, such as nasal obstruction, nasal congestion, and post-nasal drip. We were also unable to determine the motivations behind these searches. Further precise data or a big data analysis may be needed to improve the reliability of using internet search query data as a surveillance method. Third, the increase in search behaviors may have been a result of social propaganda, events, and media reports. Fourth, the peak incidence age of the three conditions varies. For example, adenoid hypertrophy is usually a condition that affects children, whereas rhinosinusitis typically affects adults; therefore, these conditions are not likely to occur in the same person. Finally, the users of search engines are mainly young, possible leading to age bias.

According to the China Statistical Report on Search Engine in 2019, Internet search engines have been widely used; $81.3 \%$ of Chinese netizens used search engines to obtain information. It was estimated that $70.5 \%$ of netizens 
used a search engine to seek specialized information in the field of medicine and/or law [30]. Surveillance systems based on the internet could compensate for the lack of clinical data, thus providing a novel option for monitoring disease morbidity and investigating the relationships among diseases.

In conclusion, $\mathrm{AH}$ and rhinosinusitis shared similar seasonal patterns, which were not consistent with AR. Reduction in the incidence of public gatherings effectively reduced the morbidities of $\mathrm{AH}$ and rhinosinusitis but not that of AR.

Author contributions All authors contributed to the study conception and design. Data collection and analysis were performed by YY. The first draft of the manuscript was written by YY and all authors commented on previous versions of the manuscript. All authors read and approved the final manuscript.

Funding This study is supported by the National Natural Science Foundation (81974142).

Availability of data and material The Baidu Index is publicly available at https://index.baidu.com.

Code availability SPSS Statistics version 26.0 is available online (https://www.ibm.com/support/pages/downloading-ibm-spss-stati stics-26).

\section{Declarations}

Conflict of interest The authors declare that they have no conflict of interest.

Ethics approval Ethics committee approval was not required for this study because it was based on Internet search data.

Consent to participate The study was based on Internet search data, and no personal information was revealed, thereby maintaining confidentiality.

\section{References}

1. Ersu R, Arman AR, Save D, Karadag B, Karakoc F, Berkem M, Dagli E (2004) Prevalence of snoring and symptoms of sleepdisordered breathing in primary school children in Istanbul. Chest 126(1):19-24. https://doi.org/10.1378/chest.126.1.19

2. Bulfamante AM, Saibene AM, Felisati G, Rosso C, Pipolo C (2019) Adenoidal disease and chronic rhinosinusitis in childrenis there a link. J Clin Med 8:10. https://doi.org/10.3390/jcm81 01528

3. Tuncer U, Aydogan B, Soylu L, Simsek M, Akcali C, Kucukcan A (2004) Chronic rhinosinusitis and adenoid hypertrophy in children. Am J Otolaryngol 25(1):5-10. https://doi.org/10.1016/j. amjoto.2003.10.003

4. Modrzynski M, Zawisza E (2007) The influence of birch pollination on the adenoid size in children with intermittent allergic rhinitis. Int J Pediatr Otorhinolaryngol 71(7):1017-1023. https:// doi.org/10.1016/j.ijporl.2007.02.018
5. Eren E, Arslanoğlu S, Erdem SB, Nacaroğlu T, Karkıner C, Can D, Önal K (2015) Chicken or the egg: the dilemma of allergic rhinitis versus adenoid hypertrophy. Rhinology 53(2):154-159. https://doi.org/10.4193/Rhin14.013

6. Ameli F, Brocchetti F, Tosca MA, Schiavetti I, Ciprandi G (2014) Tonsil volume and allergic rhinitis in children. Allergy Rhinol (Providence) 5(3):137-142. https://doi.org/10.2500/ar. 2014.5.0095

7. He G, Chen Y, Chen B, Wang H, Shen L, Liu L, Suolang D, Zhang B, Ju G, Zhang L, Du S, Jiang X, Pan Y, Min Z (2018) Using the Baidu search index to predict the incidence of HIV/ AIDS in China. Sci Rep 8(1):9038. https://doi.org/10.1038/ s41598-018-27413-1

8. Ingram DG, Matthews CK, Plante DT (2015) Seasonal trends in sleep-disordered breathing: evidence from Internet search engine query data. Sleep Breath 19(1):79-84. https://doi.org/ 10.1007/s11325-014-0965-1

9. Ingram DG, Plante DT (2013) Seasonal trends in restless legs symptomatology: evidence from Internet search query data. Sleep Med 14(12):1364-1368. https://doi.org/10.1016/j.sleep. 2013.06.016

10. Yang A, Huang N, Peng C, Tsai S (2010) Do seasons have an influence on the incidence of depression? The use of an internet search engine query data as a proxy of human affect. PLoS ONE 5(10):e13728. https://doi.org/10.1371/journal.pone.0013728

11. Ginsberg J, Mohebbi MH, Patel RS, Brammer L, Smolinski MS, Brilliant L (2009) Detecting influenza epidemics using search engine query data. Nature 457(7232):1012-1014. https://doi. org/10.1038/nature07634

12. Cao YJ, Xu R, Tao XY, Shi JB, Chen FH (2019) Investigation of temporal and spatial characteristics of allergic rhinitis epidemics by Baidu Index in China. Zhonghua Er Bi Yan Hou Tou Jing Wai Ke Za Zhi 54(12):888-893. https://doi.org/10.3760/cma.j. issn.1673-0860.2019.12.002

13. Li K, Liu M, Feng Y, Ning C, Ou W, Sun J, Wei W, Liang H, Shao Y (2019) Using Baidu search engine to monitor aids epidemics inform for targeted intervention of HIV/AIDS in China. Sci Rep 9(1):320. https://doi.org/10.1038/s41598-018-35685-w

14. Dogru M, Evcimik MF, Calim OF (2017) Does adenoid hypertrophy affect disease severity in children with allergic rhinitis? Eur Arch Otorhinolaryngol 274(1):209-213. https://doi.org/10. 1007/s00405-016-4196-x

15. Modrzynski M, Zawisza E (2007) An analysis of the incidence of adenoid hypertrophy in allergic children. Int J Pediatr Otorhinolaryngol 71(5):713-719. https://doi.org/10.1016/j.ijporl.2006. 12.018

16. Huang SW, Giannoni C (2001) The risk of adenoid hypertrophy in children with allergic rhinitis. Ann Allergy Asthma Immunol 87(4):350-355. https://doi.org/10.1016/s1081-1206(10)62251-x

17. Ameli F, Brocchetti F, Tosca M, Signori A, Ciprandi G (2013) Adenoidal hypertrophy and allergic rhinitis: is there an inverse relationship? Am J Rhinol Allergy 27(1):e5-10. https://doi.org/ 10.2500/ajra.2013.27.3854

18. Colavita L, Miraglia Del Giudice M, Stroscio G, Visalli C, Alterio T, Pidone C, Pizzino MR, Arrigo T, Chimenz R, Salpietro C, Cuppari C (2015) Allergic rhinitis and adenoid hypertrophy in children: is adenoidectomy always really useful? J Biol Regul Homeost Agents 29(2 Suppl 1):58-63

19. Lou Z (2018) Adenoid hypertrophy in children and allergic rhinitis. Eur Arch Otorhinolaryngol 275(3):831-832. https://doi. org/10.1007/s00405-017-4737-y

20. Lee D, Rosenfeld RM (1997) Adenoid bacteriology and sinonasal symptoms in children. Otolaryngol Head Neck Surg 116(3):301-307. https://doi.org/10.1016/s0194-59989770264-x 
21. Belcher R, Virgin F (2019) The role of the adenoids in pediatric chronic rhinosinusitis. Med Sci (Basel). https://doi.org/10.3390/ medsci7020035

22. Bernstein JM, Dryja D, Murphy TF (2001) Molecular typing of paired bacterial isolates from the adenoid and lateral wall of the nose in children undergoing adenoidectomy: implications in acute rhinosinusitis. Otolaryngol Head Neck Surg 125(6):593-597. https://doi.org/10.1067/mhn.2001.120232

23. Subtil J, Rodrigues JC, Reis L, Freitas L, Filipe J, Santos A, Macor C, Duarte A, Jordao L (2017) Adenoid bacterial colonization in a paediatric population. Eur Arch Otorhinolaryngol 274(4):19331938. https://doi.org/10.1007/s00405-017-4493-z

24. Fokkens WJ, Lund VJ, Mullol J, Bachert C, Alobid I, Baroody F, Cohen N, Cervin A, Douglas R, Gevaert P, Georgalas C, Goossens H, Harvey R, Hellings P, Hopkins C, Jones N, Joos G, Kalogjera L, Kern B, Kowalski M, Price D, Riechelmann H, Schlosser R, Senior B, Thomas M, Toskala E, Voegels R, de Wang Y, Wormald PJ (2012) EPOS 2012: European position paper on rhinosinusitis and nasal polyps 2012. A summary for otorhinolaryngologists. Rhinology 50(1):1-12. https://doi.org/10.4193/Rhino50E2

25. Makary CA, Ramadan HH (2013) The role of sinus surgery in children. Laryngoscope 123(6):1348-1352. https://doi.org/10. 1002/lary.23961

26. Vlastarakos PV, Fetta M, Segas JV, Maragoudakis P, Nikolopoulos TP (2013) Functional endoscopic sinus surgery improves sinusrelated symptoms and quality of life in children with chronic rhinosinusitis: a systematic analysis and meta-analysis of published interventional studies. Clin Pediatr (Phila) 52(12):10911097. https://doi.org/10.1177/0009922813506489

27. Dave D, Friedson A, Matsuzawa K, Sabia J (2020) When do shelter-in-place orders fight COVID-19 Best? Policy heterogeneity across states and adoption time. Econ Inq 15:14. https://doi.org/ 10.1111/ecin. 12944

28. Brienen NC, Timen A, Wallinga J, van Steenbergen JE, Teunis PF (2010) The effect of mask use on the spread of influenza during a pandemic. Risk Anal 30(8):1210-1218. https://doi.org/10.1111/j. 1539-6924.2010.01428.x

29. Stutt R, Retkute R, Bradley M, Gilligan C, Colvin J (2020) A modelling framework to assess the likely effectiveness of facemasks in combination with "lock-down" in managing the COVID-19 pandemic. Proc Math Phys Eng Sci 476(2238):20200376. https:// doi.org/10.1098/rspa.2020.0376

30. China Internet Network Information Center (CNNIC) (2019) China statistical report on search engine; 2019. Available from: http://www.cnnic.net.cn/hlwfzyj/. Accessed 25 Oct 2019

Publisher's Note Springer Nature remains neutral with regard to jurisdictional claims in published maps and institutional affiliations. 